\title{
Total-Body Quantitative Parametric Imaging of Early Kinetics of ${ }^{18}$ F-FDG
}

\author{
Tao Feng ${ }^{1}$, Yizhang Zhao ${ }^{2}$, Hongcheng $\mathrm{Shi}^{3}$, Hongdi $\mathrm{Li}^{1}$, Xuezhu Zhang ${ }^{4}$, Guobao Wang ${ }^{4}$, Patricia M. Price ${ }^{5}$, \\ Ramsey D. Badawi ${ }^{4,6}$, Simon R. Cherry ${ }^{4,6}$, and Terry Jones ${ }^{6}$ \\ ${ }^{1}$ UIH America Inc., Houston, Texas; ${ }^{2}$ United Imaging Healthcare, Shanghai, China; ${ }^{3}$ Zhongshan Hospital, Fudan University, \\ Shanghai, China; ${ }^{4}$ Department of Biomedical Engineering, University of California Davis, Davis, California; ${ }^{5}$ Imperial College \\ London, London, United Kingdom; and ${ }^{6}$ Department of Radiology, University of California Davis Medical Center, Davis, California
}

\begin{abstract}
Parametric imaging has been shown to provide better quantitation physiologically than SUV imaging in PET. With the increased sensitivity from a recently developed total-body PET scanner, whole-body scans with higher temporal resolution become possible for dynamic analysis and parametric imaging. In this paper, we focus on deriving the parameter $k_{1}$ using compartmental modeling and on developing a method to acquire whole-body ${ }^{18}$ F-FDG PET parametric images using only the first $90 \mathrm{~s}$ of the postinjection scan data with the total-body PET system. Methods: Dynamic projections were acquired with a time interval of $1 \mathrm{~s}$ for the first $30 \mathrm{~s}$ and $\mathrm{a}$ time interval of $2 \mathrm{~s}$ for the following minute. Image-derived input functions were acquired from the reconstructed dynamic sequences in the ascending aorta. A 1-tissue-compartment model with 4 parameters $\left(k_{1}, k_{2}\right.$, blood fraction, and delay time) was used. A maximum-likelihood-based estimation method was developed with the 1-tissue-compartment model solution. The accuracy of the acquired parameters was compared with the ones estimated using a 2-tissue-compartment irreversible model with 1-h-long data. Results: All 4 parametric images were successfully calculated using data from 2 volunteers. By comparing the time-activity curves acquired from the volumes of interest, we showed that the parameters estimated using our method were able to predict the time-activity curves of the early dynamics of ${ }^{18}$ F-FDG in different organs. The delay-time effects for different organs were also clearly visible in the reconstructed delay-time image with delay variations of as large as $40 \mathrm{~s}$. The estimated parameters using both $90-\mathrm{s}$ data and 1-h data agreed well for $k_{1}$ and blood fraction, whereas a large difference in $k_{2}$ was found between the $90-\mathrm{s}$ and 1 -h data, suggesting $k_{2}$ cannot be reliably estimated from the $90-\mathrm{s}$ scan. Conclusion: We have shown that with total-body PET and the increased sensitivity, it is possible to estimate parametric images based on the very early dynamics after ${ }^{18} \mathrm{~F}-\mathrm{FDG}$ injection. The estimated $k_{1}$ might potentially be used clinically as an indicator for identifying abnormalities.
\end{abstract}

Key Words: parametric imaging; early dynamic; total-body PET; compartment model

J Nucl Med 2021; 62:738-744

DOI: 10.2967/jnumed.119.238113

Received Nov. 13, 2019; revision accepted Aug. 6, 2020.

For correspondence or reprints contact: Tao Feng, UIH America, Inc., 9230 Kirby Dr, Suite 600, Houston, TX 77054.

E-mail: tao.feng@united-imaging.com

Published online Sep. 18, 2020.

COPYRIGHT (C 2021 by the Society of Nuclear Medicine and Molecular Imaging.
C onventional PET imaging makes widespread use of SUVs for semiquantitative analysis, especially in measurements of ${ }^{18} \mathrm{~F}$ FDG uptake in tumors (1). However, SUV depends on the time of measurement and many other factors $(2,3)$, which makes reliable quantitation a challenging task. On the other hand, kinetic analysis in PET has more direct physiologic meaning than does SUV and can be a more robust approach for quantifying ${ }^{18} \mathrm{~F}-\mathrm{FDG}$ uptake and use $(4,5)$.

Compared with volume-of-interest (VOI)-based kinetic analysis $(6,7)$, parametric imaging enables the acquisition of voxel-level dynamics of tracer uptake by applying kinetic modeling for each individual voxel $(8,9)$. Conventional graphical methods, including Logan analysis (10) and Patlak analysis (11), have been used for parametric imaging because of their linear properties and simplicity in acquisition protocols. Improved lesion contrast and detectability have been demonstrated by many studies $(12,13)$. With the assumption that the dynamic activity can be described using exponentials as impulse responses, the basis function provides a possible approach for nonlinear model fitting $(14,15)$. Recently, whole-body parametric imaging using the Patlak model has been proposed using a multipass acquisition protocol $(16,17)$.

Even with the many added benefits of kinetic modeling, parametric imaging techniques and dynamic protocols are not widely used in clinical studies, especially in oncology (17). In some studies, the requirement for invasive arterial blood sampling for the input function makes the scan protocol impractical. Even with image-derived input functions $(18,19)$ or population-based input functions $(20,21)$, a minimal scan time of $30 \mathrm{~min}$ is often required to reveal dynamic information using Patlak analysis. Compared with state-of-the-art whole-body SUV scans, which take around $20 \mathrm{~min}$ and sometimes less than $10 \mathrm{~min}$, the increased scan time and more complex protocol have proved to be one of the major practical factors that hinder the clinical use of dynamic analysis with whole-body imaging.

The study of early dynamics, such as first-pass studies $(22,23)$, on the other hand, may enable much shorter scan protocols and-with ${ }^{18}$ F-FDG studies - may enable the estimation of $k_{1}$, which represents the rate of ${ }^{18} \mathrm{~F}-\mathrm{FDG}$ transport from the arterial plasma to the intracellular space. In some studies in the literature, ${ }^{18} \mathrm{~F}$-FDG $k_{1}$ was found to be an indicator for identifying tumor subgroups (24), gene expressions (25), and chemotherapy response (26) and could be used to assess tumor blood flow (22). ${ }^{18}$ F-FDG $k_{1}$ was found to be a valuable parameter for applications other than oncology. In the work 
by Sarkar et al. (27), the authors found that the $k_{1}$ value is helpful for quantifying liver inflammation in nonalcoholic fatty liver disease.

With the recently developed total-body PET system, a dramatic improvement in sensitivity has made it possible to acquire PET images with reasonable quality in scan times of $60 \mathrm{~s}$ or less $(28,29)$. With this increase in sensitivity, parametric imaging with high temporal resolution becomes feasible, which in turn may allow accurate parametric imaging using only the early phases of the tracer dynamics. We have developed a method to acquire whole-body parametric images using data acquired from the total-body PET system with a temporal resolution of 1-2 s. With the much improved temporal resolution, the delay time of the input function has been shown to be an important factor in VOI-based analysis (30). In this paper, the delay time is included as a voxel-specific parameter that is jointly estimated.

\section{MATERIALS AND METHODS}

\section{Parametric Image Reconstruction}

For ${ }^{18} \mathrm{~F}$-FDG, a 2-tissue-compartment irreversible (2Ti) model with 4 rate constants $\left(k_{1}, k_{2}, k_{3}\right.$, and $\left.k_{4}\right)$ is often used to model its tracer dynamics. In the 2Ti model, $k_{1}$ and $k_{2}$ describe the forward and backward transport of ${ }^{18} \mathrm{~F}$-FDG from plasma to tissue. $k_{3}$ and $k_{4}$ model the phosphorylation and dephosphorylation process. The accuracy of the measured kinetic parameters in dynamic analysis can be estimated by calculating the sensitivity of the model with respect to each kinetic parameter (31). Zuo et al. (32) suggested that $k_{3}$ and $k_{4}$ cannot be accurately estimated using only the first few minutes of a scan because of the low identifiability of these parameters at early times. Therefore, a 1-tissue-compartment (1T) model was used in this study. The timeactivity curve for a specific voxel can be expressed as...

$$
C(t)=v_{b} C_{b}(t)+\left(1-v_{b}\right)\left(k_{1} \exp \left(-k_{2} t\right) \otimes C_{p}(t)\right),
$$

where $v_{b}$ is the blood fraction in the tissue, $C_{b}(t)$ is the time-activity curve for the whole blood, $C_{p}(t)$ is the plasma input function, and $C(t)$ is the concentration of ${ }^{18} \mathrm{~F}-\mathrm{FDG}$ in the tissue. $\otimes$ represents the convolution operation. In the above equation, the whole-blood time-activity curve is used for the blood fraction because the intravascular activity includes that from both plasma and blood cells.

With higher temporal resolution and whole-body coverage, the delay time for different regions becomes an important factor. With the inclusion of delay time, the measured time-activity curve is...

$$
C(t)=v_{b} C_{b}\left(t-t_{d}\right)+\kappa_{1} \exp \left(-k_{2} t\right) \otimes C_{p}\left(t-t_{d}\right),
$$

Eq. 2

where $t_{d}$ is the voxel-dependent delay time. For easier computation, the parameter $\kappa_{1}=\left(1-v_{b}\right) k_{1}$ is used and estimated instead of $k_{1}$.

Because of the very high temporal resolution used in this study, the time-activity curve for an individual voxel is still expected to be noisy despite the much-increased sensitivity of the scanner. Therefore, it may be beneficial to develop a maximum-likelihood estimation approach that specifically models the noise of the reconstructed dynamic image. Studies in the literature indicate that the voxel values in the image approximately follow a scaled Poisson distribution (33). With this assumption, the log-likelihood function can be derived as...

$$
L\left(\kappa_{1}, k_{2}, v_{b}, t_{d}\right)=\sum_{t}-C(t)+x_{t} \log (C(t))-\log \left(x_{t} !\right),
$$

where $x_{t}$ are the reconstructed dynamic images and are treated as a known measurement in this case. The update equation for $\kappa_{1}, k_{2}$, and $v_{b}$ can be derived by maximizing the likelihood in the above equation

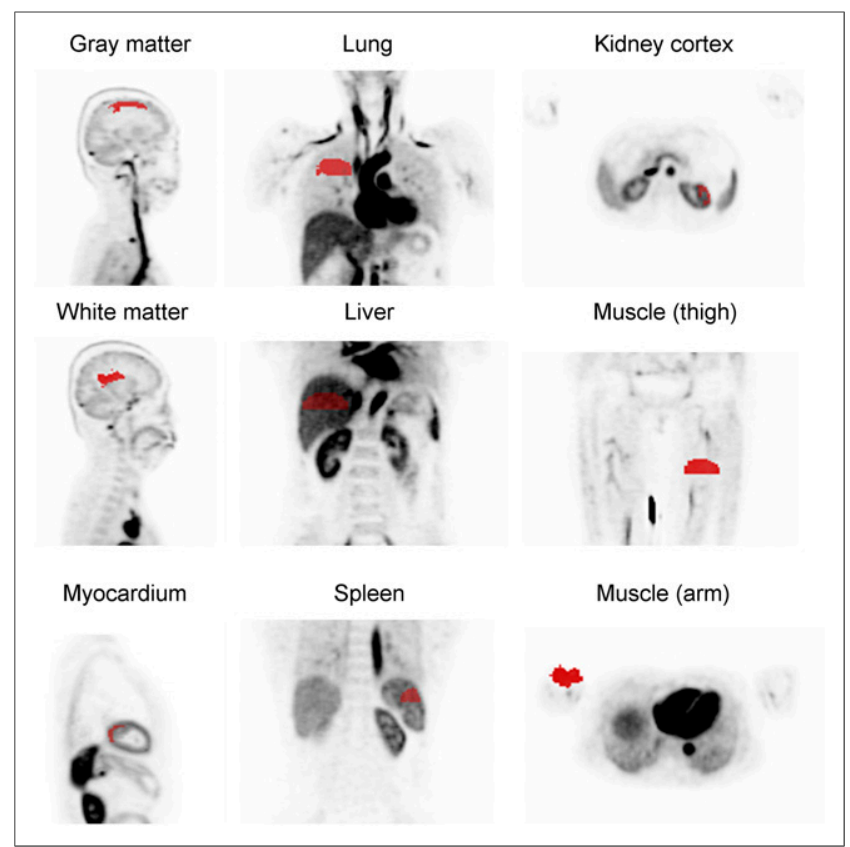

FIGURE 1. VOls used for parameter estimation.

(Supplemental Eqs. 1-5 show the derivations; supplemental materials are available at http://jnm.snmjournals.org):

$$
\left\{\begin{array}{l}
\boldsymbol{v}_{b}^{p+1}=\frac{\boldsymbol{v}_{b}^{p}}{\sum_{t} C_{b}\left(t-\boldsymbol{t}_{d}\right)} \sum_{t} \frac{C_{b}\left(t-\boldsymbol{t}_{d}\right) \boldsymbol{C}(t)}{\hat{\boldsymbol{C}}^{p}(t)} \\
\kappa_{1}^{p+1}=\frac{\kappa_{1}^{p}}{\sum_{t} e^{-k_{2}^{p} t} \otimes C_{p}\left(t-\boldsymbol{t}_{d}\right)} \sum_{t} \frac{e^{-k_{2}^{p} t} \otimes C_{p}\left(t-\boldsymbol{t}_{d}\right) \boldsymbol{C}(t)}{\hat{\boldsymbol{C}}^{p}(t)} \\
k_{2}^{p+1}=k_{2}^{p} \frac{\sum_{t} t \kappa_{1}^{p}\left(\exp \left(-k_{2}^{p} t\right)\right) \otimes C_{p}\left(t-\boldsymbol{t}_{\boldsymbol{d}}\right)}{\sum_{t} \frac{\kappa_{1}^{p}\left(\exp \left(-k_{2}^{p} t\right)\right) \otimes C_{p}\left(t-\boldsymbol{t}_{\boldsymbol{d}}\right) \boldsymbol{C}(t)}{\hat{\boldsymbol{C}}^{p}(t)}}
\end{array}\right.
$$

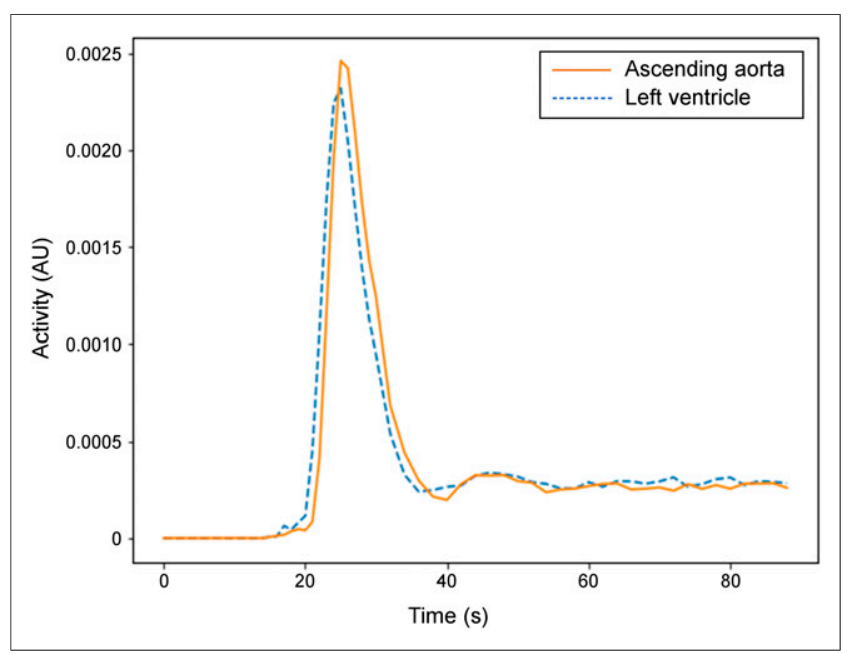

FIGURE 2. Image-derived input function for first $90 \mathrm{~s}$ after injection. $\mathrm{AU}=$ arbitrary units. 


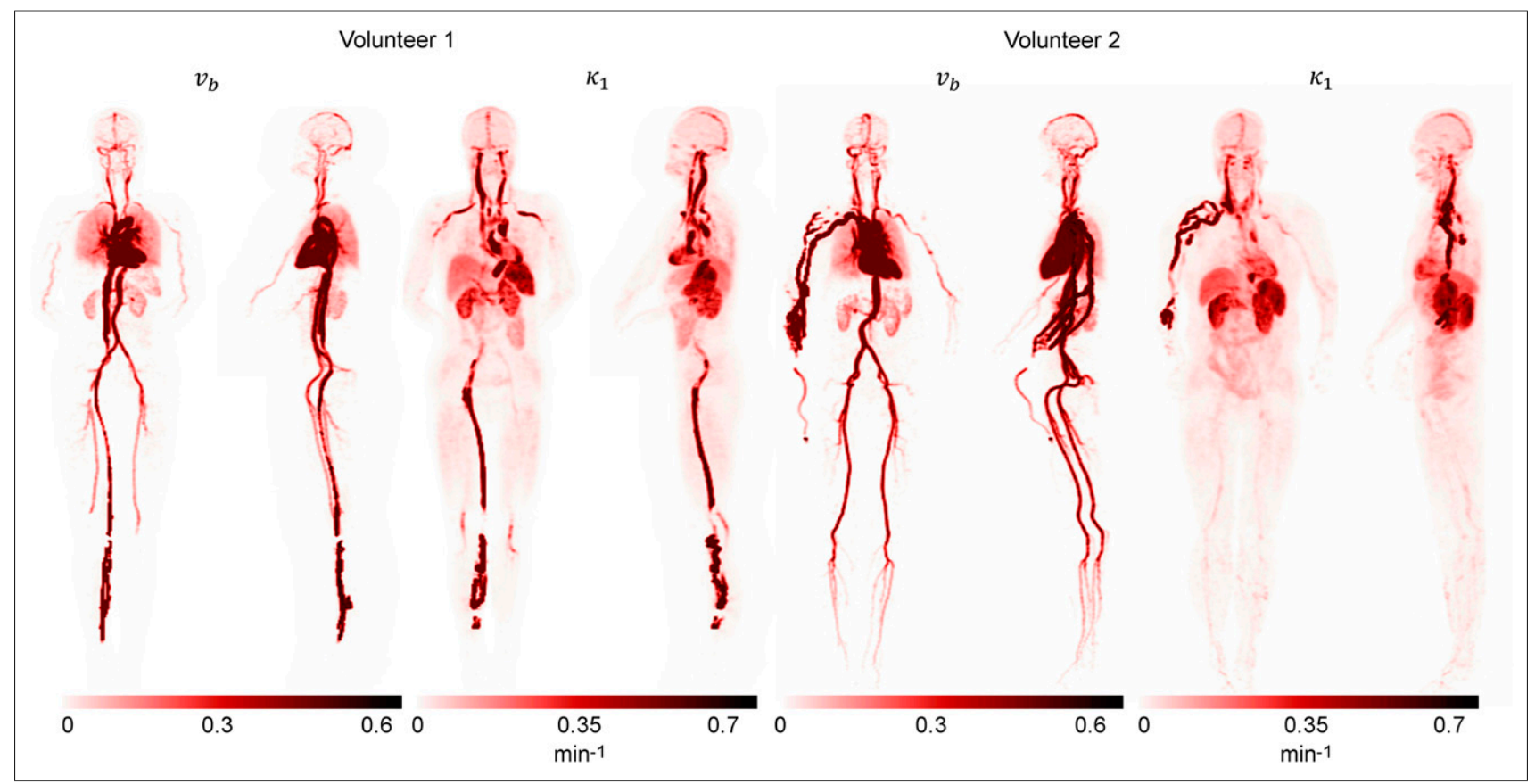

FIGURE 3. Maximum-intensity projection of reconstructed parametric images $\boldsymbol{v}_{b}$ and $k_{1}$ acquired using proposed approach.

where $p$ is the iteration number and $\hat{C}^{p}(t)=v_{b}^{p} C_{b}\left(t-t_{d}\right)+\kappa_{1}^{p} e^{-k_{2}^{p} t} \otimes$ $C_{p}\left(t-t_{d}\right)$ are the estimated dynamic images. $\hat{\boldsymbol{C}}^{p}$ is the matrix version of $\hat{C}^{p}$; the same is true for the other symbols as well. The multiplicative update equations imply positive constraint for $\kappa_{1}, k_{2}$, and $v_{b}$, which is reasonable on the basis of the model. The multiplicative factors for $\kappa_{1}$ and $v_{b}$ are similar to that from a conventional maximum-likelihood expectation maximization approach to image reconstruction, as is the reciprocal of $k_{2}$ 's update factor. This similarity makes the implementation straightforward.
A maximum-likelihood gradient-descent approach is used for the estimation of $t_{d}$ (Supplemental Eq. 6 shows the derivations):

$$
t_{d}^{p+1}=t_{d}^{p}+s \frac{\partial L\left(\kappa_{1}, k_{2}, v_{b}, t_{d}\right)}{\partial t_{d}},
$$

where $\frac{\partial L\left(\kappa_{1}, k_{2}, v_{b}, t_{d}\right)}{\partial t_{d}}=\sum_{t}\left(v_{b} C_{b}^{\prime}\left(t-t_{d}\right)-\kappa_{1} \exp \left(-k_{2} t\right) \otimes C_{p}^{\prime}\left(t-t_{d}\right)\right)$ $\left(\frac{x_{t}}{\hat{c}^{\prime}(t)}-1\right)$, and $s$ is the step size of the update. Since the range of

delay times is expected to be less than $1 \mathrm{~min}$ based on known circulation times, to ensure convergence the absolute update for the delay time was set to a fixed value for all voxels in this paper, that is, $s=\Delta t /\left|\frac{\partial L\left(\kappa_{1}, k_{2}, v_{b}, t_{d}\right)}{\partial t_{d}}\right|$. The update value $\Delta t$ was set to be $2 \mathrm{~s}$ in the first few iterations and reduced to $0.2 \mathrm{~s}$ in later iterations.

In this study, the dynamic images were first reconstructed by applying 3 iterations with 20 subsets using time-of-flight information. The reconstruction voxel size was $4 \times 4 \times$ $2.89 \mathrm{~mm}$, and attenuation, scatter, randoms, and normalization corrections and pointspread-function modeling were also included during image reconstruction. A 3-dimensinal gaussian filter of $6 \mathrm{~mm}$ in full width at half maximum was used for noise suppression in the reconstructed dynamic image. An alternate update approach was used to estimate the 4 parameters. In total, 18 main iterations were used. Within each iteration, $\kappa_{1}$ and $\boldsymbol{v}_{b}$ were first updated with fixed $\boldsymbol{k}_{2}$ and $\boldsymbol{t}_{d}$ using 9 subiterations, $\boldsymbol{k}_{2}$ was updated with fixed $\boldsymbol{\kappa}_{1}$, $\boldsymbol{v}_{b}$, and $\boldsymbol{t}_{d}$ using 1 subiteration, followed by 
TABLE 1

Estimated $k_{1}$ and Delay Time (Relative to Ascending Aorta) in Different Organs

\begin{tabular}{|c|c|c|c|c|c|c|c|c|c|}
\hline Parameter & Gray matter & White matter & Myocardium & Liver & Kidney cortex & Spleen & Lung & Arm & Thigh \\
\hline $\begin{array}{l}k_{1}\left(\min ^{-1}\right) \text { VOI } \\
\text { analysis }\end{array}$ & 0.08 & 0.05 & 0.56 & 0.39 & 0.75 & 0.92 & 0.02 & 0.02 & 0.06 \\
\hline $\begin{array}{l}k_{1}\left(\min ^{-1}\right) \\
\quad \text { parametric } \\
\text { image }\end{array}$ & $0.09 \pm 0.02$ & $0.06 \pm 0.01$ & $0.45 \pm 0.10$ & $0.33 \pm 0.04$ & $0.54 \pm 0.08$ & $0.73 \pm 0.13$ & $0.02 \pm 0.02$ & $0.02 \pm 0.01$ & $0.06 \pm 0.03$ \\
\hline$C_{b}$ VOI analysis & 0.020 & 0.01 & 0.02 & 0.006 & 0.08 & 0.006 & 0.12 & 0.0004 & 0.0 \\
\hline $\begin{array}{l}C_{b} \text { parametric } \\
\text { image }\end{array}$ & $0.020 \pm 0.01$ & $0.007 \pm 0.005$ & $0.05 \pm 0.04$ & $0.003 \pm 0.005$ & $0.11 \pm 0.03$ & $0.03 \pm 0.02$ & $0.12 \pm 0.03$ & $0.0009 \pm 0.001$ & $0.004 \pm 0.01$ \\
\hline Delay time (s) & 3.9 & 3.7 & -1.2 & 13.1 & 4.7 & 3.1 & -3.8 & 10.8 & 8.6 \\
\hline
\end{tabular}

the update of $\boldsymbol{t}_{d}$ with fixed $\kappa_{1}, \boldsymbol{v}_{b}$, and $\boldsymbol{k}_{2}$ using 3 subiterations. The same gaussian smoothing filter was used after every 6 main iterations for the delay-time image and $\kappa_{1}$ for better control of noise. With the acquired $\kappa_{1}$ and $v_{b}$ image, the $k_{1}$ image was also generated and compared with $\kappa_{1}$.

To study the accuracy of the estimated parameters, they were compared with VOI-based analysis. Nine regions were selected (gray matter, white matter, lung, liver, kidney cortex, myocardium, spleen, arm, and thighs; Fig. 1). A 2 Ti model was also applied on 1-h-long data to compare the estimated $k_{1}$ values. In the $2 \mathrm{Ti}$ model, the same delay time as estimated using the $1 \mathrm{~T}$ model was used because the extended time is less likely to contain information on the delay time.

\section{Patient Data and Scan Protocol}

Two healthy volunteers with an injected activity of about $220 \mathrm{MBq}$ were scanned using the uExplorer PET scanner (United Imaging Healthcare) with the approval of the Ethics Committee of Zhongshan Hospital and after giving informed consent. One volunteer was injected in the leg, and the other was injected in the arm. The image data obtained during the first $90 \mathrm{~s}$ after injection were used in this study for $1 \mathrm{~T}$ model analysis.

To obtain an image-derived input function, VOIs over the ascending aorta and the left ventricle (Supplemental Fig. 1) were manually drawn on the PET/CT image and the time-activity curves were acquired. It has been suggested that the best location for image-derived input function is the ascending aorta (34), as it is less affected by respiratory and cardiac motion. The left ventricle is another widely used location because of the relatively large blood pool size (18). The diameter of the ascending aorta VOI was $16 \mathrm{~mm}$, and the height was $40 \mathrm{~mm}$. The boundaries in the left ventricle were excluded from the left ventricular VOI to reduce the effect of respiratory motion, and the total volume was about the same as for the ascending aorta VOI.
Whole-blood-to-plasma correction was applied on the time-activity curve $C_{b}(t)$ to estimate the plasma input function $C_{p}(t)$. The correction equations and values are based on the method and parameters published in the literature (Eq. 2 of Wahl et al. (35), where the hematocrit is 0.42 and the equilibration time constant is $0.23 \mathrm{~min}^{-1}$ ).

\section{RESULTS}

Figure 2 shows the first $90 \mathrm{~s}$ of the image-derived plasma input function for volunteer 1 using the reconstructed dynamic images of the ascending aorta VOI and the left ventricular VOI. Image voxel values were applied directly without calibration of activity concentrations using a well counter. The calibration scale factor cancels out in the analysis and therefore is not needed. The timeactivity curves were almost identical except for the 1-s delay time. With the added delay time modeling, the results are expected to be the same with the left ventricle as the input function. The timeactivity curve from the ascending aorta was used for the input function.

Figure 3 shows the acquired maximum-intensity projection from the reconstructed $\kappa_{1}$ and $v_{b}$ images. The reconstructed parametric images $v_{b}, \kappa_{1}, k_{2}$, and $t_{d}$ from volunteer 1 are shown in Figure 4. The $k_{1}$ image was also calculated using $v_{b}$ and $\kappa_{1}$. The SUV image acquired from the same periods (0-90 s) is also shown for comparison. $v_{b}$ represents the plasma volume fraction in the tissue, which was clearly visible in the blood pool of the heart and in the aorta. The visual difference between $k_{1}$ and $\kappa_{1}$ was generally small, except for the myocardium region. High $k_{1}$ values were observed in the spleen and kidneys. Moderate $k_{1}$ values were observed in the liver and myocardium. A high $k_{1}$ was also observed in some blood vessels (mostly veins), likely the result of dispersion of the plasma
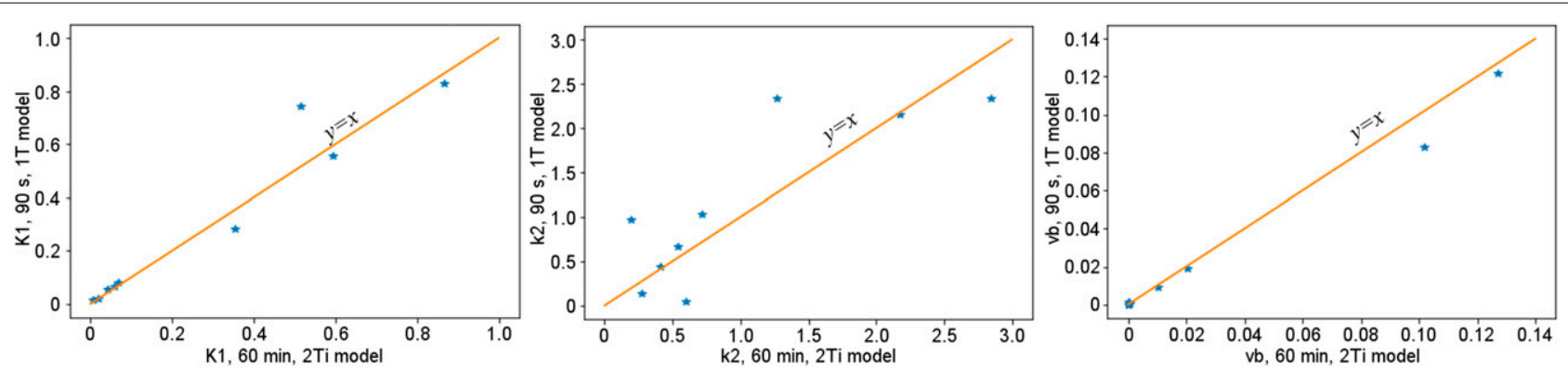

FIGURE 5. Estimated $k_{1}, k_{2}$, and $v_{b}$ for 9 VOls using $1 \mathrm{~T}$ model with $90 \mathrm{~s}$ of data (y-axis) and 2Ti model with $1 \mathrm{~h}$ of data ( $x$-axis). 


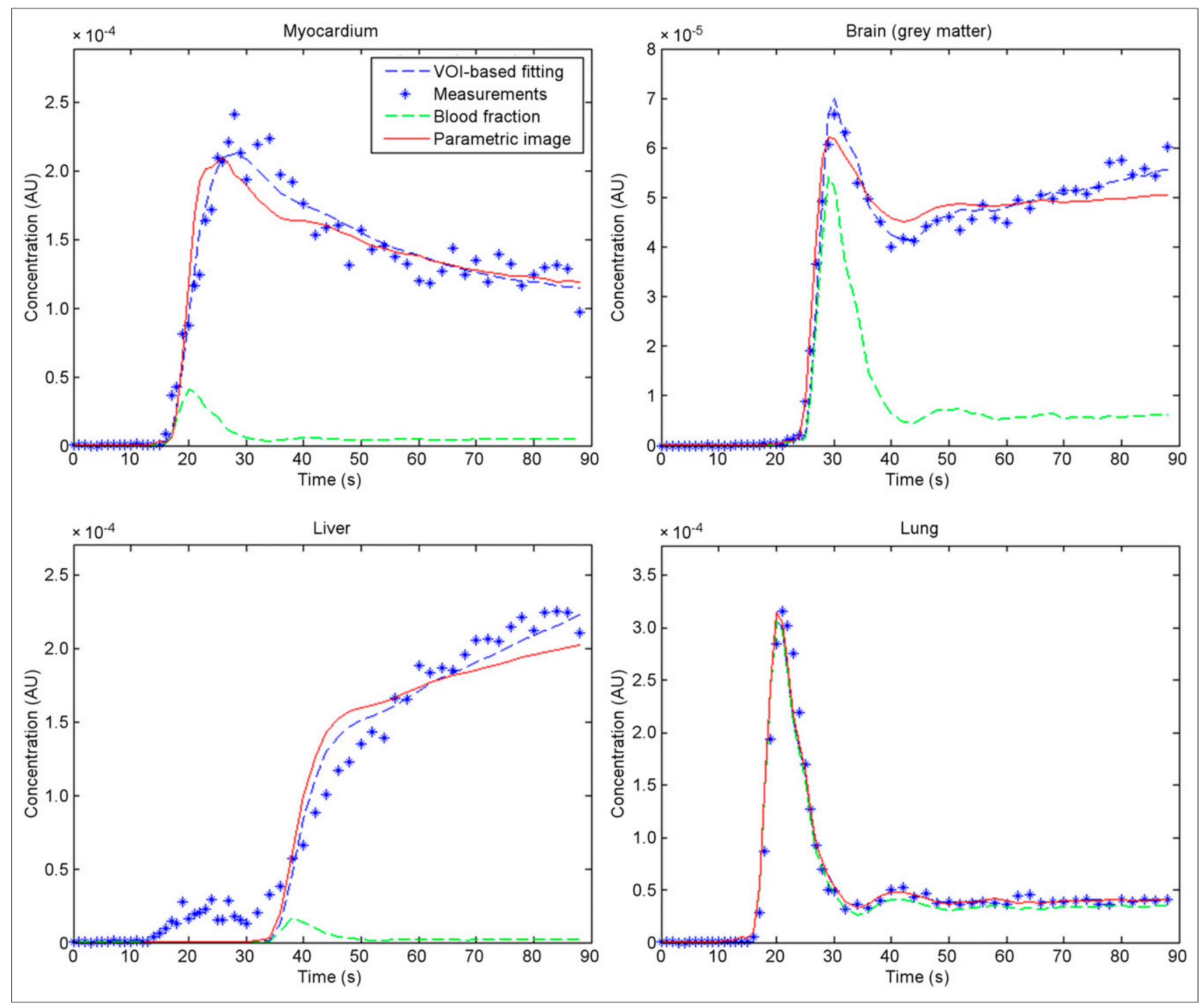

FIGURE 6. Time-activity curve measured from dynamic frames (blue dots) and fitted curves using proposed method, and fitting based on timeactivity curve from VOI in 4 different organs: myocardium, brain, liver, and lung. For better illustration of delay time, corresponding blood fraction in time-activity curve was also plotted. $\mathrm{AU}=$ arbitrary units.

input function in these vessels. The abnormal signal in the veins near the injection site suggested possible retrograde pooling in the veins. Very large $k_{2}$ values were also observed in some blood vessels for the same reason. Large delay times were observed in the liver $(>10 \mathrm{~s})$ and extremities $(\sim 30 \mathrm{~s})$ when compared with the delay time in the lungs. The large delay time in the liver region was likely caused by the dual blood supply from both the hepatic artery and the portal vein (36), and the algorithm approximated the portal vein input function with the arterial input function by means of a delay time. The delay-time image could also be used for the illustration of the circulatory system. In volunteer $1,{ }^{18} \mathrm{~F}-\mathrm{FDG}$ was first injected into a leg vein and then traveled to the right ventricle. From there the tracer was moved into the lungs and back to the left ventricle, where the tracer was pumped via the arterial system to other organs. The measured delay times (relative to the ascending aorta), $k_{1}$, and $v_{b}$ for different organs are given in Table 1 .

Using VOI analysis, the estimated $k_{1}, k_{2}$, and blood fraction $v_{b}$ using the 1T model with $90 \mathrm{~s}$ of data were compared with the same parameters calculated using the 2Ti model with 1-h-long data. Figure 5 shows the relationships of the estimated values. For $k_{1}$ and $v_{b}$, both approaches yielded similar results (coefficient of determination of linear fitting was 0.97 for $k_{1}$ and 0.95 for blood fraction). Some large differences were observed in $k_{2}$, suggesting that $k_{2}$ cannot be accurately estimated from the 90 -s data. The estimated $k_{1}$ using VOI-based analysis was also compared with the $k_{1}$ acquired from the parametric image and is given in Table 1. For most VOIs, the differences in $k_{1}$ were small. $k_{1}$ was underestimated in VOIs in the myocardium and kidney cortex, likely because of application of the gaussian filter, which introduced additional partial-volume effects and affected the quantitative values within these relatively small structures.

Figure 6 shows the time-activity curves for myocardium, brain, liver, and lung. The average parameters within the VOI were used to estimate the fitted curves. Because the ascending aorta was used as the input function, a positive delay time was observed in the liver and a negative delay time in the myocardium and lung, with 
a minimal delay time in the brain. The difference between the curves from averaged voxel-based parameters and from the VOIbased parameters was relatively small, indicating a small bias from the voxel-based analysis. Even though the model used in this paper was generally able to predict the time-activity curve from different organs with high temporal resolution, discrepancies could be observed in some organs such as the liver.

\section{DISCUSSION}

This preliminary study showed that the $k_{1}$ estimated using 90-s data generally agrees with that derived from a much longer scan $(1 \mathrm{~h})$. The same agreement was not found for $k_{2}$, indicating that the estimated $k_{2}$ is less accurate in shorter scans. High $k_{1}$ values were detected in some venous blood pools, such as in the jugular veins, venous sinuses, and portal veins, whereas high blood fractions were observed in arterial blood pools such as the carotid arteries (Fig. 3). The high $k_{1}$ values in some blood-pool regions were likely caused by the dispersion effects modeled by the $1 \mathrm{~T}$ model (37). This information may yield further physiologic insights and better kinetic modeling. For instance, the $k_{1}$ in the venous blood pools of the brain may represent the combined effects of the glucose passing through the brain's vasculature and the small extraction of tracers within the brain. The $k_{1}$ in the portal veins may also be used to develop a more accurate model for the liver. The modeling of the delay times in the kinetic model not only enabled better model fitting but also produced a delaytime image that might provide additional information-for example, as a biomarker for assessing blood delivery and vessel occlusion.

However, our method did not model the dispersion effects of the input function for different organs. Another limitation of the proposed approach is the use of a single model for the whole body. For instance, the dual blood supplies (portal veins and arteries) in the liver region (32) were not modeled. The use of a single input function resulted in some quantitative inaccuracy, and further studies are required for more accurate parametric imaging. The image-based input function in this study was also not validated using arterial blood samples, but the feasibility has already been proven in the literature (18). Because of the very concentrated and rapidly changing tracer distribution in the first few seconds after injection, scatter corrections may not be accurate enough. One possible example is the low delay time in regions near the injection site in the delay-time image. Another example is the small increase in the liver time-activity curve around $20 \mathrm{~s}$ after injection, which we believe was caused by the inaccurate scatter correction from the very high activity level during the injection.

Two different injection sites were used in our study. Initially the leg was chosen so that the injection site would be closer to the end of the gantry, thus requiring a shorter tube connecting the syringe and the needle; however, a major challenge in leg injection is the difficulty in finding the appropriate vein. Subsequent to the first patient, we chose to perform all injections in the antecubital fossa, which follows conventional practice and has not led to significant difficulties (38).

Although our method was developed using ${ }^{18}$ F-FDG, the same methodology can be applied to other tracers as well. More benefit can be expected for radionuclides with a much shorter half-life, such as ${ }^{15} \mathrm{O}$ or ${ }^{82} \mathrm{Rb}$. The acquired ${ }^{18} \mathrm{~F}$-FDG $k_{1}$ image can also be used to examine the relationship between blood flow and metabolism, which may help with diagnosis in some pathophysiologic conditions such as hyperemic areas of brain tissue after stroke. For ${ }^{18}$ F-FDG scans, a practical implementation of the proposed approach can still be challenging, as ${ }^{18} \mathrm{~F}-\mathrm{FDG}$ imaging traditionally focuses mostly on the later period, when equilibrium is reached. Combination with a later scan may be the solution; however, future studies are required to address the clinical or technical challenges for that protocol.

Future studies using patient data are required for a better understanding of the full potential of the clinical significance of the proposed method.

\section{CONCLUSION}

In this study, we demonstrated that with an ultra-high-sensitivity total-body PET scanner, it is possible to achieve whole-body parametric image reconstruction using only the early stage of the scan (within the first 2 min after injection), for much easier incorporation into the daily clinical route. However, only fast parameters such as $k_{1}$ can be estimated using this ultrashort scan duration. We also showed that with the much-improved temporal resolution due to improved sensitivity, organ-dependent delay time becomes an important factor to consider in the analysis of whole-body early-stage dynamics.

\section{DISCLOSURE}

Tao Feng and Hongdi Li are employed by UIH America, Inc., and Yizhang Zhao is employed by United Imaging Healthcare. Simon R. Cherry and Ramsey D. Badawi have a research agreement with United Imaging Healthcare. The University of California Davis has a revenue-sharing agreement with United Imaging Healthcare. This work was partially supported by the National Institutes of Health under grant R01 CA206187. No other potential conflict of interest relevant to this article was reported.

\section{KEY POINTS}

QUESTION: Can whole-body first-pass parametric imaging be achieved using an ultrashort scanning protocol?

PERTINENT FINDINGS: We have demonstrated the possibility of generating whole-body parametric images such as $k_{1}$ (a possible indicator for tumor identification), blood fraction, and delay-time images that illustrate the circulatory system, from dynamic scans of as short as $90 \mathrm{~s}$ using the total-body PET scanner.

IMPLICATIONS FOR PATIENT CARE: This protocol produces added value (a potential biomarker for identification of tumors and other diseases) with minimal added scanner time.

\section{REFERENCES}

1. Lindholm P, Minn H, Leskinen-Kallio S, Bergman J, Ruotsalainen U, Joensuu H. Influence of the blood glucose concentration on FDG uptake in cancer: a PET study. J Nucl Med. 1993;34:1-6.

2. Adams MC, Turkington TG, Wilson JM, Wong TZ. A systematic review of the factors affecting accuracy of SUV measurements. AJR. 2010;195:310-320.

3. Boellaard R. Need for standardization of ${ }^{18}$ F-FDG PET/CT for treatment response assessments. J Nucl Med. 2011;52(suppl):93S-100S.

4. Hamberg LM, Hunter GJ, Alpert NM, Choi NC, Babich JW, Fischman AJ. The dose uptake ratio as an index of glucose metabolism: useful parameter or oversimplification? J Nucl Med. 1994;35:1308-1312.

5. Nesterov SV, Deshayes E, Sciagrà R, et al. Quantification of myocardial blood flow in absolute terms using ${ }^{82} \mathrm{Rb}$ PET imaging: the RUBY-10 study. JACC Cardiovasc Imaging. 2014;7:1119-1127.

6. Muzi M, Vesselle H, Grierson JR, et al. Kinetic analysis of $3^{\prime}$-deoxy-3'-fluorothymidine PET studies: validation studies in patients with lung cancer. $J$ Nucl Med. 2005;46:274-282.

7. Gulaldi N, Xia J, Feng T, et al. Modeling of the renal kinetics of the AT1 receptor specific PET radioligand $\left[{ }^{11} \mathrm{C}\right]$ KR31173. BioMed Res Int. 2013;2013:835859. 
8. Kamasak ME, Bouman CA, Morris ED, Sauer K. Direct reconstruction of kinetic parameter images from dynamic PET data. IEEE Trans Med Imaging. 2005;24:636-650.

9. Zhang X, Xie Z, Berg E, et al. Total-body dynamic reconstruction and parametric imaging on the uEXPLORER. J Nucl Med. 2020;61:285-291.

10. Logan J. Graphical analysis of PET data applied to reversible and irreversible tracers. Nucl Med Biol. 2000;27:661-670.

11. Patlak CS, Blasberg RG. Graphical evaluation of blood-to-brain transfer constants from multiple-time uptake data: generalizations. J Cereb Blood Flow Metab. 1985;5:584-590.

12. Wu H, Dimitrakopoulou-Strauss A, Heichel TO, et al. Quantitative evaluation of skeletal tumours with dynamic FDG PET: SUV in comparison to Patlak analysis. Eur J Nucl Med. 2001;28:704-710.

13. Visser EP, Philippens MEP, Kienhorst L, et al. Comparison of tumor volumes derived from glucose metabolic rate maps and SUV maps in dynamic ${ }^{18} \mathrm{~F}-\mathrm{FDG}$ PET. J Nucl Med. 2008;49:892-898.

14. Cunningham VJ, Jones T. Spectral analysis of dynamic PET studies. J Cereb Blood Flow Metab. 1993;13:15-23.

15. Watabe H, Jino H, Kawachi N, et al. Parametric imaging of myocardial blood flow with ${ }^{15} \mathrm{O}$-water and PET using the basis function method. J Nucl Med. 2005;46:1219-1224.

16. Karakatsanis NA, Lodge MA, Tahari AK, Zhou Y, Wahl RL, Rahmim A. Dynamic whole-body PET parametric imaging: I. Concept, acquisition protocol optimization and clinical application. Phys Med Biol. 2013;58:7391-7418.

17. Rahmim A, Lodge MA, Karakatsanis NA, et al. Dynamic whole-body PET imaging: principles, potentials and applications. Eur J Nucl Med Mol Imaging. 2019;46:501-518.

18. de Geus-Oei L-F, Visser EP, Krabbe PFM, et al. Comparison of image-derived and arterial input functions for estimating the rate of glucose metabolism in therapy-monitoring ${ }^{18}$ F-FDG PET studies. J Nucl Med. 2006;47:945-949.

19. Feng T, Tsui BMW, Li X, et al. Image-derived and arterial blood sampled input functions for quantitative PET imaging of the angiotensin II subtype 1 receptor in the kidney. Med Phys. 2015;42:6736-6744.

20. Takikawa S, Dhawan V, Spetsieris P, et al. Noninvasive quantitative fluorodeoxyglucose PET studies with an estimated input function derived from a population-based arterial blood curve. Radiology. 1993;188:131-136.

21. Rissanen E, Tuisku J, Luoto $\mathrm{P}$, et al. Automated reference region extraction and population-based input function for brain $\left[{ }^{11} \mathrm{C}\right]$ TMSX PET image analyses. $J$ Cereb Blood Flow Metab. 2015;35:157-165.

22. Mullani NA, Herbst RS, O’Neil RG, Gould KL, Barron BJ, Abbruzzese JL. Tumor blood flow measured by PET dynamic imaging of first-pass ${ }^{18} \mathrm{~F}-\mathrm{FDG}$ uptake: a comparison with ${ }^{15}$ O-labeled water-measured blood flow. J Nucl Med. 2008;49:517-523.

23. Cochet A, Pigeonnat S, Khoury B, et al. Evaluation of breast tumor blood flow with dynamic first-pass ${ }^{18} \mathrm{~F}-\mathrm{FDG}$ PET/CT: comparison with angiogenesis markers and prognostic factors. J Nucl Med. 2012;53:512-520.
24. Sugawara Y, Zasadny KR, Grossman HB, Francis IR, Clarke MF, Wahl RL. Germ cell tumor: differentiation of viable tumor, mature teratoma, and necrotic tissue with FDG PET and kinetic modeling. Radiology. 1999;211:249-256.

25. Cheng C, Klippel S, Koczan D, Willis S, Pan L, Sachpekidis C. Gene expression profiling of colorectal cancer by correlation with ${ }^{18} \mathrm{~F}-\mathrm{FDG}$ kinetics as measured by dynamic positron emission tomography-computed tomography (dPET-CT): dependency on cadherin-related genes and hypoxia. Clin Oncol. 2017;2:1179.

26. Song SL, Deng C, Wen L, et al. ${ }^{18}$ F-FDG PET/CT-related metabolic parameters and their value in early prediction of chemotherapy response in a VX2 tumor model. Nucl Med Biol. 2010;37:327-333.

27. Sarkar S, Corwin MT, Olson KA, et al. Pilot study to diagnose nonalcoholic steatohepatitis with dynamic ${ }^{18}$ F-FDG PET. AJR. 2019;212:529-537.

28. Cherry SR, Badawi RD, Karp JS, Moses WW, Price P, Jones T. Total-body imaging: transforming the role of positron emission tomography. Sci Transl Med. 2017;9:eaaf6169.

29. Badawi RD, Shi $\mathrm{H}, \mathrm{Hu}$ P, et al. First human imaging studies with the EXPLORER total-body PET scanner. J Nucl Med. 2019;60:299-303.

30. Lammertsma AA, Cunningham VJ, Deiber MP, et al. Combination of dynamic and integral methods for generating reproducible functional CBF images. $J$ Cereb Blood Flow Metab. 1990;10:675-686.

31. Mankoff DA, Muzi M, Zaidi H. Quantitative analysis in nuclear oncologic imaging. In: Quantitative Analysis in Nuclear Medicine Imaging. Springer; 2006: 494-536.

32. Zuo Y, Sarkar S, Corwin MT, Olson KA, Badawi RD, Wang G. Structural and practical identifiability of dual-input kinetic modeling in dynamic PET of liver inflammation. Phys Med Biol. 2019;64:175023.

33. Wilson DW, Tsui BMW, Barrett HH. Noise properties of the EM algorithm. II. Monte Carlo simulations. Phys Med Biol. 1994;39:847-871.

34. van der Weerdt AP, Klein LJ, Boellaard R, Visser CA, Visser FC, Lammertsma AA. Image-derived input functions for determination of MRGlu in cardiac ${ }^{18} \mathrm{~F}-\mathrm{FDG}$ PET scans. J Nucl Med. 2001;42:1622-1629.

35. Wahl LM, Asselin M-C, Nahmias C. Regions of interest in the venous sinuses as input functions for quantitative PET. J Nucl Med. 1999;40:1666-1675.

36. Munk OL, Bass L, Roelsgaard K, Bender D, Hansen SB, Keiding S. Liver kinetics of glucose analogs measured in pigs by PET: importance of dual-input blood sampling. J Nucl Med. 2001;42:795-801.

37. Iida H, Kanno I, Miura S, Murakami M, Takahashi K, Uemura K. Error analysis of a quantitative cerebral blood flow measurement using $\mathrm{H}_{2}{ }^{15} \mathrm{O}$ autoradiography and positron emission tomography, with respect to the dispersion of the input function. J Cereb Blood Flow Metab. 1986;6:536-545.

38. McBride K, Hunt HL. Bolus injection technique for uEXPLORER ${ }^{18} \mathrm{~F}-\mathrm{FDG}$ PET/CT dynamic scans [abstract]. J Nucl Med. 2020;61(suppl 1):3084. 